\title{
A comment on psychological distress and women's hair loss related to the COVID-19 pandemic
}

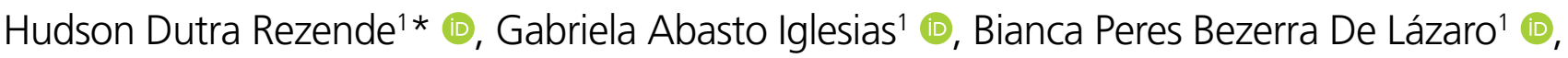 \\ Rafael Vidigal de Abreu Morais ${ }^{2}\left[10\right.$, Sandra Lopes Mattos Dinato ${ }^{1}{ }^{\circ}$, \\ Maria Fernanda Reis Gavazzoni Dias ${ }^{3}$
}

The COVID-19 pandemic has brought many uncertainties as to its origins and future directions ${ }^{1}$. While scientists of different specialties focus on new strategies on how to minimize physical sequels related to COVID-19 infection, many patients remain vulnerable to having their psychological state neglected.

In 2020, inevitable doubts have emerged, as the pandemic developed, impacting the patients' quality-of-life not just by the restrictions imposed by the governmental efforts to halt virus spread worldwide, but also by the unpredictable behaviour of COVID-19, such as its infectivity, pathogenicity, virulence, lethality, and immunogenicity ${ }^{2}$.

From that timepoint onwards, as social contacts reduced and due to the patients' mourning for their family members death, health practitioners were invited to look at their patients from a more comprehensive perspective, caring for both physical and psychological aspects.

The study of early psychological aspects during the pandemic, especially for women, has been published by many authors, generating interesting data. The first paper reporting the psychological impact of the COVID-19 outbreak came from Spain, where González-Sanguino and colleagues conducted a survey with 3,480 people and found that female patients were more likely to show greater depressive symptoms and anxiety ${ }^{3}$. Accordingly, Choi et al. evaluated the level of depression and anxiety in 500 patients from Hong Kong, where $25.4 \%$ of the respondents reported that their mental health had deteriorated since the COVID-19 pandemic began ${ }^{4}$, reinforcing the importance of González-Sanguino's work.
It is true that long before the pandemic, hair loss was already considered a major source of suffering for female patients requiring dermatological assistance. In accordance with Trüeb, as stated in his book "The difficult hair loss patient", hair loss pictures one of the most genuine concerns presented by women of several ages in dermatology, irrespective of other concomitant skin conditions ${ }^{5}$. Nonetheless, hair-loss related complaints are still prone to be neglected in clinical practice.

The importance of hair for women's psychological status is gradually attracting attention from different specialties, and patients under extremely stressful situations have shown concern for their hair, even when facing life-threatening conditions. For instance, in 2017, Villar et al. prospectively evaluated 339 patients at the Breast Cancer Unit of the University Hospital Complex of A Coruña (Spain) who had been undergoing treatment for breast cancer from December 2013 to February $2015^{6}$. Hair loss was cited by the patients as one of the most disturbing consequences of chemotherapy during the whole process ${ }^{6}$.

Although it is not known yet the impact of associated hairloss related stress and pandemic-related anxiety on women's quality-of-life, the number of patients complaining of hair problems has recently increased, since several types of alopecia can be worsened by physical and psychological distress ${ }^{1,7,8}$. Accordingly, the number of reports on alopecia areata and telogen effluvium, either aggravated or induced by COVID-19 infection, constantly rises in the literature ${ }^{7,8}$.

It is possible that the severity of the hair shed correlates with the severity of COVID-19 infection ${ }^{1}$. Patients with high fever

\footnotetext{
${ }^{1}$ Centro Universitário Lusíada, Departamento de Dermatologia - Santos (SP), Brazil.

Universidade do Grande Rio Professor José de Souza Herdy - Rio de Janeiro (RJ), Brazil.

${ }^{3}$ Universidade Federal Fluminense, Departamento de Dermatologia - Rio de Janeiro (RJ), Brazil.

${ }^{*}$ Corresponding author: contato@hudsondutra.com.br

Conflicts of interest: the authors declare there is no conflicts of interest. Funding: none.

Received on June 22, 2021. Accepted on June 27, 2021.
} 
seem to experience more hair loss than asymptomatic ones ${ }^{1}$. It is interesting to notice that post COVID-19 telogen effluvium develops earlier than expected for general febrile conditions and can be rather intense, bringing extra source of anxiety for the affected women ${ }^{1}$. Also, alopecia areata, a common dermatological source of distress for many patients worldwide, seem to have increased during the pandemic too, though it is not clear whether the virus itself is implicated in the pathogenesis or if the recent cases only reflect the new levels of psychological stress peculiarly seen during the pandemic.

In fact, it is not only because COVID-19 infection has eventually been proven that every hair problem can be regarded as a mere consequence. It is likely to think that patients who were receiving regular treatment for any given alopecia might have gotten worse, since lack of accessibility to hospitals and restrictions to medications has built barriers to proper assistance.

Finally, as the pandemic develops and COVID-19 complications appear, it becomes difficult for the attending physician to wisely deal with every patient's need. As previously exposed, the new scenario favours the appearance of mental and physical illness, urging health practitioners to look for a multidisciplinary approach. As for many other medical areas, specialized care is also needed when evaluating patients complaining of hair loss.

\section{AUTHORS" CONTRIBUTIONS}

HDR: Conceptualization, Formal analysis, Supervision, Validation, Visualization, Writing - original draft, Writing - review \& editing. GAI: Conceptualization, Data curation, Formal analysis, Validation, Visualization, Writing - original draft, Writing review \& editing. BPBDL: Formal analysis, Funding acquisition, Investigation, Methodology, Project administration, Resources, Writing - original draft, Writing - review \& editing. RVAM: Conceptualization, Data curation, Project administration, Resources, Supervision, Validation, Visualization, Writing - original draft, Writing - review \& editing. SLMD: Conceptualization, Data curation, Formal analysis, Methodology, Project administration, Resources, Supervision, Validation, Visualization, Writing - original draft, Writing - review \& editing. MFRGD: Data curation, Formal analysis, Project administration, Software, Supervision, Validation, Visualization, Writing - original draft, Writing - review \& editing.

\section{REFERENCES}

1. Trüeb RM, Dutra Rezende H, Gavazzoni Dias MFR. What can the hair tell us about COVID-19? Exp Dermatol. 2021;30(2):28890. https://doi.org/10.1111/exd.14259

2. Souza CDF. In times of COVID-19, epidemiology is a unifying science. Rev Assoc Med Bras (1992). 2020;66(Suppl 2):27-30. https://doi.org/10.1590/1806-9282.66.S2.27

3. González-Sanguino C, Ausín B, Castellanos MÁ, Saiz J, LópezGómez A, Ugidos C, et al. Mental health consequences during the initial stage of the 2020 coronavirus pandemic (COVID-19) in Spain. Brain Behav Immun. 2020;87:172-6. https://doi. org/10.1016/j.bbi.2020.05.040

4. Choi EPH, Hui BPH, Wan EYF. Depression and anxiety in Hong Kong during COVID-19. Int J Environ Res Public Health. 2020;17(10):3740. https://doi.org/10.3390/ijerph17103740
5. Trüeb R. The difficult hair loss patient: guide to successful management of alopecia and related conditions. Switzerland: Springer International Publishing, 2015. https://doi. org/10.1007/978-3-319-19701-2_2

6. Villar RR, Fernández SP, Garea CC, Pillado MTS, Barreiro VB, Martín CG. Quality of life and anxiety in women with breast cancer before and after treatment. Rev Lat Am Enfermagem. 2017;25:e2958. https://doi.org/10.1590/1518-8345.2258.2958

7. Flvenson D. COVID-19: association with rapidly progressive forms of alopecia areata. Int J Dermatol. 2021;60(1):127. https://doi.org/10.1111/ijd.15317

8. Mieczkowska K, Deutsch A, Borok J, Guzman AK, Fruchter R, Patel $P$, et al. Telogen effluvium: a sequela of COVID-19. Int J Dermatol. 2021;60(1):122-4. https://doi.org/10.1111/ijd.15313 